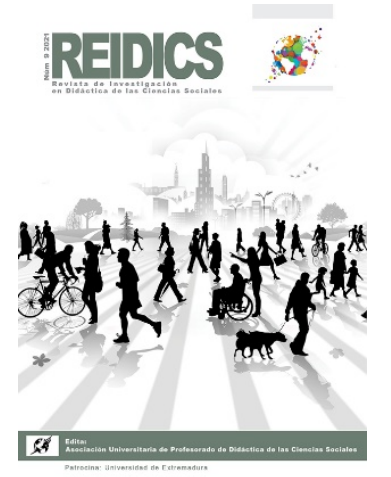

\title{
REIDICS
}

Revista de Investigación en

Didáctica de las Ciencias

Sociales
Núm. 9, 2021

Recibido 06 mayo 2021

Aceptado 30 setiembre 2021

E-ISSN: 2531-0968

\section{La enseñanza de problemas socio-ambientales. Reflexiones para la innovación educativa*}

\section{Teaching socio-environmental problems. Reflections for educational innovation}

Carlos Fuster García

Universitat de València

Email: carlos.fuster-garcia@uv.es

ORCID: https://orcid.org/0000-0002-1180-0391

Diego García Monteagudo

Universitat de València

Email: Diego.Garcia-Monteagudo@uv.es

ORCID: https://orcid.org/0000-0003-0505-0608

Xosé Manuel Souto González

Universitat de València

Email: Xose.Manuel.Souto@uv.es

ORCID: https://orcid.org/0000-0003-1480-327X

DOI: https://doi.org/10.17398/2531-0968.09.79

\section{Resumen}

En los dos decenios finales del siglo XX se generó un debate sobre las posibilidades de mejorar la enseñanza de las ciencias sociales desde el tratamiento de los problemas sociales y ambientales. Los principales grupos de innovación presentaron propuestas de formación del profesorado y elaboración de materiales y metodologías para llevar adelante estas iniciativas. Algunos grupos, como GREDICS, IRES y Gea-Clío, han continuado con estos programas de trabajo, que se han denominado de diferentes maneras: problemas socialmente relevantes, cuestiones socialmente vivas, temas socialmente controvertidos, no siempre coincidentes en sus finalidades. Sin embargo, su aplicación en los currículos escolares es escasa, tanto por problemas de formación docente como por las suspicacias ideológicas que aparecen en el seno de la comunidad escolar. En este artículo nos remontamos a los antecedentes del siglo XX, donde formulamos un proyecto curricular con estos presupuestos. Más adelante analizamos los resultados obtenidos en la investigación educativa en las relaciones entre problemas sociales y escolares, un camino de ida y vuelta que muestra la pertinencia de esta metodología 
para favorecer una actitud participativa ciudadana por parte de las personas que ejercen el rol de alumnos y alumnas. Una implicación social que no sólo afecta al alumnado, sino también al resto de agentes educativos: profesorado, familias y administración educativa, pues los resultados del trabajo escolar afectan a la vida cotidiana.

Palabras clave: Problemas sociales relevantes; representaciones sociales; grupos de innovación; participación ciudadana.

\begin{abstract}
In the final two decades of the 20th century, a debate was generated about the possibilities of improving the teaching of social sciences from the treatment of social and environmental problems. The main innovation groups presented proposals for teacher training and preparation of materials and methodologies to carry out these initiatives. Some groups, such as GREDICS, IRES and GEA-CLÍO, have continued with these work programs, which have been called in different ways: socially relevant problems, socially alive questions, socially controversial issues, not always coinciding in their purposes. However, its application in school curricula is scarce, both due to teacher training problems and the ideological suspicions that appear within the school community. In this article we go back to the antecedents of the 20th century, where we formulated a curricular project with these assumptions. Later we analyse the results obtained in educational research on the relationships between social and school problems, a round trip that shows the relevance of this methodology to promote a participatory citizen attitude on the part of the people who play the role of students and female students. A social implication that not only affects students, but also the rest of educational agents: teachers, families and educational administration, since the results of school work affect daily life.
\end{abstract}

Key words: Relevant social problems; social representations; innovation groups; citizen participation.

\title{
1. Introducción
}

Desde hace más de treinta años estamos trabajando en la necesidad de mejorar el aprendizaje del alumnado en el área de ciencias sociales, geografía e historia. Nuestra posición implica modificar el clásico temario de hechos conceptuales que deben aprender los alumnos/as por un modelo educativo que suponga la selección de contenidos educativos que permitan analizar los principales problemas, desde los ámbitos del tiempo histórico y el espacio geográfico, que condicionan nuestra comprensión del mundo (Souto, García-Monteagudo y Fuster, 2016).

Pero dicha propuesta es una tarea compleja y supone entender cómo actúa el conocimiento humano para explicar los hechos sociales. En el caso del saber escolar nos hemos aproximado a este asunto con un estudio de las percepciones y representaciones del alumnado, que tenía problemas para entender la cultura hegemónica que se transmite de forma tradicional en los centros escolares (Souto, García-Monteagudo y Fuster, 2018). Sostenemos así una teoría interpretativa que relaciona las expectativas personales con las condiciones institucionales que definen la construcción de un nuevo conocimiento escolar, que está condicionado por la representación social de la educación básica.

En este artículo pretendemos seguir el programa de investigación que hemos trazado desde hace años, pero que se ha complementado con la aportación de las teorías de las representaciones 
sociales, en especial los conceptos clave de "sentido práctico" y "sentido común", donde las aportaciones de los clásicos (Pierre Bourdieu o Serge Moscovici) han sido reinterpretadas con la ayuda de M. Domingos (Domingos, 2000; Domingos y Diniz, 2019).

Entendemos que el colectivo docente de los niveles de educación básica en España (desde los 3 a 18 años de edad del alumnado) está sometido a unas reglas institucionales que le condicionan su manera de decidir. Por eso, el sentido práctico determina las actuaciones del profesorado ante el comportamiento del alumnado, las quejas ante la falta de compromiso y su actitud para estudiar o la falta de competencia teórica. Dicha necesidad de actuar de forma rápida y automática (sentido práctico) justifica el sentido común del profesorado, que se corresponde con las rutinas y tradiciones que se han criticado por los grupos de innovación y que causan insatisfacción a docentes y alumnos.

Nuestra aportación quiere ejemplificar dos casos de posibles actuaciones escolares. Por un lado, queremos mostrar cómo los estudios desde las aulas escolares pueden promover la participación social para resolver problemas que afectan no sólo a la comunidad escolar, sino a más personas. Por otra parte, hemos considerado algunos problemas existentes más allá de los límites del ámbito educativo escolar, para ver cómo se pueden trasladar a las aulas para ser sintetizados como contenidos escolares. No es algo nuevo, pues los antecedentes de esta forma de trabajar guardan semejanzas con propuestas realizadas por la Escuela Moderna, hace más de cien años. Nosotros utilizaremos ejemplos más recientes, del último cuarto de siglo XX en España, donde los grupos de innovación mostraban sus deseos de mejorar una docencia con la que mostraban claras discrepancias. Finalizamos el trabajo con la presentación de unas conclusiones hipotéticas, que son el resultado de verificar con evidencias empíricas documentales y de observación de clases, la dialéctica entre la problemática social y escolar.

\section{Antecedentes de la investigación escolar}

Como miembros de Gea-Clío, nuestras finalidades han estado condicionadas por el contexto de las transformaciones del sistema escolar en España, que respondía a muchos de los deseos de los grupos de innovación por mejorar la enseñanza de las ciencias sociales en las etapas de la educación básica. Con posterioridad a la implantación de la Ley Orgánica General del Sistema Educativo (LOGSE), uno de los debates más relevantes ha sido el referido a la organización de los contenidos curriculares en el área de las ciencias sociales. Se establecía, entonces, una dicotomía entre problemas y disciplinas (Cronos, 1997), como si no fuera posible abordar los problemas sociales básicos desde el conocimiento disciplinar, que se asemejaba a una concepción tradicional y singular. Se importaron nuevas perspectivas de organización del conocimiento escolar, que estaban vinculadas a las tradiciones de los socials studies de Estados Unidos (problemas socialmente relevantes) y a los intentos de renovación de la enseñanza de las ciencias sociales, entre ellas de la geografía e historia en el ámbito francófono (cuestiones socialmente vivas). Son aspectos de una búsqueda de alternativas a la enseñanza de las ciencias sociales que nos remiten a planteamientos teóricos anteriores, tanto en el campo de la pedagogía (Dewey, Decroly, Kilpatrick) como en el de la epistemología (Antiseri, Morin). 
De esta manera se estaban definiendo algunos conceptos, que conviene diferenciar. En primer lugar, los problemas socialmente relevantes, generalmente de ámbito interdisciplinario (Ocampo y Valencia, 2019), que tenía uno de sus ejemplos más representativos en el grupo andaluz IRES con su referencia a los conceptos metadisciplinares y a las teorías de Edgar Morin:

La cuestión nos la hemos de plantear, pues, ante todo, en el marco de la selección, organización y formulación de los contenidos de enseñanza. Más concretamente, entendemos que lo fundamental al respecto sería establecer criterios que faciliten la selección de objetos de estudio relativos a problemas de nuestro mundo (es decir, de tópicos, centros de interés, problemáticas de investigación escolar...) que tengan mayor potencialidad para integrar contenidos educativos relevantes (interpretaciones del mundo, conceptos específicos, destrezas educativas, sistemas de valores). (Merchán y García Pérez, 1997, p. 61)

En segundo lugar, las cuestiones socialmente vivas, desde el ámbito de la didáctica de las ciencias sociales en grupos como Aula Sete o Cronos. Una posición que se mantiene en el caso de la formación del profesorado en el colectivo de la Universitat Autónoma de Barcelona, como se muestra a continuación:

Es reivindica un currículum basat en problemes socials, en el desenvolupament d'una consciència històrica, i en la formació d'un pensament social crític que permeti als nois i a les noies en-frontar-se als conflictes i als problemes i que els faci competents per voler participar en la construcció del futur. (Pagès i Santisteban, 2011, p. 9)

Y tercero, los temas controvertidos en la enseñanza de las ciencias sociales. En este caso el grupo GREDICS de Barcelona, con una propuesta de raíz historiográfica. Se busca no sólo explicar el pasado, sino elaborar juicios de valor éticos sobre problemas que admiten diferentes interpretaciones y no se han cerrado. Un modelo muy ligado a la historiografía norteamericana y con conexiones teóricas con la denominada literacidad crítica (González y Santisteban, 2020).

Dichas diferencias no nos deben alejar de nuestros propósitos iniciales, como es la definición de un problema social que se pueda explicar escolarmente. En este sentido hemos considerado la definición de problema de la Real Academia Española (RAE) de 2020: "Planteamiento de una situación cuya respuesta desconocida debe obtenerse a través de métodos científicos", que nosotros transformamos en la búsqueda de una metodología, de un camino, para encontrar una respuesta que sea creíble para poder adoptar soluciones en la vida cotidiana. La búsqueda de otras definiciones conceptuales en el histórico de la RAE nos permite identificar algunos rasgos relevantes para entender su uso como método didáctico.

En la definición de 1780 nos dice que un problema es una "qüestion (sic) que regularmente se propone para exercitar (sic) el ingenio y se puede defender afirmativa y negativamente con razones en pro y en contra". En el Diccionario de 1832 se añade en esta entrada "la propuesta que se hace de investigar alguna verdad desconocida, o de practicar alguna cosa". En 1901, en plena institucionalización del sistema escolar, un problema se definía como "cuestión que se trata de aclarar, proposición dudosa”. En la segunda mitad del siglo XX encontramos su relación con las finalidades, pues se añade que es "un conjunto de hechos o circunstancias que dificultan la consecución de algún fin” (RAE, 1970). 
Es decir, desde una perspectiva de la interpretación del pasado un problema ha permitido identificar una actitud (ejercitar el ingenio), unas habilidades (utilizar razones en pro y en contra) y una proposición de actuación (investigar una verdad desconocida) con arreglo a una finalidad. Estas características son las que defendemos en nuestra posición educativa que, sin embargo, no es aceptada por la mayoría del cuerpo docente.

Esta misma percepción es la que analizan Víctor Santidrián y Ramón L. Facal, en su defensa de una práctica educativa basada en problemas sociales candentes, que ellos quieren implementar aprovechando la legislación educativa:

La legislación establece para la enseñanza de las ciencias sociales unas finalidades que justifican la introducción en el aula de temas socialmente conflictivos. Este artículo define qué se entiende por conflicto social candente y cuáles son las razones por las que presenta las diferentes actitudes del profesorado ante este tipo de temas... (Santidrián y López Facal, 2011, p. 8)

Ello nos permitirá identificar hasta qué punto la selección de problemas permite no sólo explicar cuestiones relevantes para la sociedad, sino que sobre todo facilitará el desarrollo de una actitud que cuestione la información que recibe y, más de una vez, satura la capacidad de razonamiento del alumnado. En este sentido hemos visto que el planteamiento y resolución de problemas facilita la formación docente (García-Monteagudo, Fuster y Souto, 2017; Ortega y Pagès, 2017; Pagès y Santisteban, 2011), un camino que hemos asumido en la organización de los contenidos de formación inicial del profesorado de Educación Secundaria.

\section{Método: análisis de fuentes para comprender el camino hasta la innovación}

Las tradiciones educativas de la Escuela Moderna ya nos señalaban la necesidad de relacionar los hechos sociales fuera de las aulas con el estudio que se realizaba en su interior. La generalización de la educación escolar a más cohortes de edad favoreció que surgieran colectivos que reforzaron dicha tendencia, en especial, en el período de la Segunda República y con conexiones entre las investigaciones educativas y su aplicación a la innovación escolar. En los años sesenta del siglo XX algunos colectivos se organizaron para recuperar esta tradición de innovación, que estaba oculta bajo el manto de tradicionalismo escolar del franquismo. La transición política a la democracia, tras la muerte de Franco, favoreció la eclosión de grupos que reivindicaban un papel de las ciencias sociales como instrumentos de compromiso con el cambio y la participación social.

La metodología que seguimos es comparativa crítica. Hemos realizado un análisis documental del conocimiento obtenido en las investigaciones. Con los resultados públicos de investigaciones y ejemplos de innovaciones ofrecidas por los grupos de innovación hemos procedido a establecer síntesis sobre las concepciones que se establecen en relación con los problemas sociales y su implicación en el conocimiento escolar. Procedemos de esta manera para mostrar la relevancia de la dialéctica entre fenómenos sociales relevantes y problemas de aprendizaje escolar. Entendemos que así se avanza en la funcionalidad ciudadana del saber escolar. 
Hemos buscado los antecedentes a esta revisión conceptual en las aportaciones de los grupos de innovación didáctica existentes en los dos decenios finales del siglo XX en España, que han sido estudiados en una Tesis doctoral reciente (Duarte, 2015). Sabemos de las dificultades de empezar el estudio desde una reflexión subjetiva, pues puede contaminar el resto del análisis, pero también le concede el refuerzo del compromiso entre el hacer y el decir.

Como se ha dicho, en los decenios finales del siglo XX esta tarea era común a diferentes grupos de innovación escolar (Asklepios, Cronos, Ínsula Barataria, IRES, Gea-Clío, Kairós...), que con propuestas teóricas y confección de materiales curriculares propiciaban una manera de trabajar más cercana a los problemas sociales cotidianos. En sus propuestas encontramos una programación de contenidos para la Secundaria Obligatoria y para el Bachillerato, que estaba muy ligada a la selección de unos problemas relevantes.

En el caso del grupo gallego Aula Sete, los contenidos relacionados con problemas del mundo actual se orientaban a partir de ocho proposiciones:

1. El sistema político de democracia parlamentaria, que se ha extendido recientemente en el mundo occidental, está basado en el respeto a los derechos y libertades individuales de las personas, en la separación de poderes y en el sufragio universal (...).

8. Las políticas socialmente avanzadas se caracterizan por los intentos de compensar las desigualdades entre los grupos sociales y ofrecer oportunidades de desarrollo a los menos favorecidos. (Pedrouzo, Díaz y López Facal, 1995)

Algo semejante a lo que exponíamos desde Gea-Clío:

1으 Programar las unidades didácticas desde problemas sociales relevantes: explicar las desigualdades ante la vida y la muerte, defender el derecho a una vivienda digna, promover la búsqueda de la paz entre Países, tener lugares donde disfrutar de nuestro tiempo libre, saber convivir con los riesgos naturales, evitar el agotamiento de los recursos naturales... Problemas que aparecen en los diferentes Decretos de Mínimos de Primaria, Secundaria y Bachillerato.

$2^{\circ}$ ) Transformar estas situaciones sociales en cuestiones problemáticas para el alumnado, de tal forma que pueda construir un camino (una metodología) que le ayude a plantear y resolver los problemas desde su propio conocimiento, enterándose de las cosas que expone y explica. (Souto y Ramírez, 1996)

Unos principios teóricos que se concretaban en una selección de Unidades Didácticas para ser desarrolladas en un proyecto curricular para toda la etapa Secundaria Obligatoria, que se difundieron en cuadernos didácticos para alumnado y docentes en los años noventa del siglo pasado. Más tarde, en el primer decenio del siglo XXI ${ }^{1}$, se han editado estos materiales en forma de manuales, manteniendo así una continuidad en nuestra argumentación de los problemas en relación con los contenidos.

La selección de problemas sociales la hemos relacionado con los principios explicativos de la geografía e historia, pues ambas materias permiten aflorar los factores temporales y espaciales que determinan la vida en sociedad. En este sentido, entendimos, por ejemplo, que la ciudad no es un problema, sino que en la ciudad hay problemas, como el de la vivienda, el de sostenibilidad o

1Se pueden consultar los cuadernos y guías docentes del proyecto Gea-Clío en la página web del grupo de investigación Social(S): http://socialsuv.org/gea-clio/ 
el de la gentrificación. Una forma semejante de interpretar el currículo escolar como la que se hace desde Andalucía, en relación con la Educación Primaria² ${ }^{2}$ en la cual se presentan los problemas sociales de participación o de recursos hídricos como eje para articular los contenidos curriculares. Dicha forma de interpretar el currículo nos ha permitido desarrollar unos contenidos básicos en relación con los problemas que queremos analizar en clase (el acceso a la vivienda, las agresiones a los equilibrios ecológicos, la identidad ciudadana). Entendemos que la investigación escolar se puede concretar en la elaboración de informes, noticias, dossiers... sobre la vida cotidiana, con clara implicación en la vida institucional local. Por eso en el siglo XXI nos hemos relacionado con el proyecto Nós Propomos ${ }^{3}$, pues ello nos facilitó la labor de difundir los problemas en los ámbitos sociales y políticos. En síntesis, hemos programado unas Unidades Didácticas que facilitaban la elaboración del conocimiento escolar desde el análisis de los problemas cotidianos. Primero se transformaron en cuadernos que se compartían con el resto de la comunidad escolar, después fueron los proyectos políticos dentro del contexto de Nosaltres Proposem en la Comunidad Valenciana.

\section{Tabla 1}

Organización de Unidades Didácticas desde los problemas sociales relevantes.

\begin{tabular}{|c|c|c|c|c|}
\hline $\begin{array}{l}\text { Problemas } \\
\text { referencia }\end{array}$ & $\begin{array}{l}\text { Conceptos } \\
\text { espaciales }\end{array}$ & $\begin{array}{l}\text { Lugares } \\
\text { geográficos }\end{array}$ & Tipo de Geografía & $\begin{array}{l}\text { Actitudes } \\
\text { Destrezas }\end{array}$ \\
\hline $\begin{array}{l}\text { 1er ciclo de } \\
\text { Secundaria: } \\
\text { ¿Cómo nos } \\
\text { identificamos con } \\
\text { un lugar? } \\
\text { ¿Por qué nacemos } \\
\text { y morimos } \\
\text { desiguales? } \\
\text { ¿Por qué hay } \\
\text { racismo y } \\
\text { xenofobia? }\end{array}$ & $\begin{array}{l}\text { Percepción } \\
\text { Comportamiento } \\
\text { espacial } \\
\text { Escalas y órdenes } \\
\text { de magnitud } \\
\text { Distribución y } \\
\text { localización } \\
\text { espacial }\end{array}$ & $\begin{array}{l}\text { Localidad } \\
\text { España } \\
\text { Lugares del } \\
\text { Mundo (en } \\
\text { diferentes } \\
\text { escalas) }\end{array}$ & $\begin{array}{l}\text { Radical, } \\
\text { Percepción y } \\
\text { Comportamiento }\end{array}$ & $\begin{array}{l}\text { Contraste de } \\
\text { percibir y } \\
\text { observar } \\
\text { Acotar y } \\
\text { representar un } \\
\text { lugar } \\
\text { Diferenciar } \\
\text { elementos y } \\
\text { lugares } \\
\text { Entender factores, } \\
\text { localizar objetos y } \\
\text { personas en un } \\
\text { lugar }\end{array}$ \\
\hline
\end{tabular}

Fuente: Souto y Ramírez, 1996.

Sin duda este planteamiento es difícil de realizar en un marco institucional de forma individual. Por eso siempre hemos defendido la idea de organizar equipos de trabajo y proyectos curriculares, donde se relacione la investigación académica con la innovación didáctica, en la cual el alumnado adquiere el protagonismo de elaborar su aprendizaje y los y las docentes desarrollan estrategias de investigación que dignifican la autoridad intelectual del profesorado de la enseñanza básica. Además, nos permite obtener evidencias de la gradación de complejidad en problemas y asuntos que se delimitan en un tiempo y en un espacio. Así lo podemos comprobar

\footnotetext{
2 Ver Orden de 10 de agosto de 2007, por la que se desarrolla el currículo correspondiente a la Educación Primaria en Andalucía: https://www.juntadeandalucia.es/boja/2007/171/1, en especial las páginas 4 a 23.

3 Nós Propomos! es un proyecto de participación ciudadana desde el análisis de problemas locales. Ha iniciado su andadura en el año 2011 desde la Universidade de Lisboa y después se ha difundido por todo Portugal, Brasil, Colombia, España, México, Mozambique y Perú.
} 
en el siguiente ejemplo (Tabla 1) de gradación de contenidos curriculares en Secundaria Obligatoria en el caso del proyecto Gea-Clío ${ }^{4}$.

\section{Resultados de la dialéctica entre problemas sociales y escolares}

Como hemos señalado en la introducción, queremos mostrar dos ejemplos dialécticos de la relación entre problemas sociales y escolares, que según el análisis que estamos realizando se pueden integrar en una explicación ciudadana. La conversión de problemas sociales en escolares supone una metodología que permita identificar el problema, para buscar información fiable que nos permita verificar las conjeturas e hipótesis que se plantean en clase y poder intervenir posteriormente en la sociedad.

\subsection{La representación social de un problema escolar. El caso del medio rural}

Las personas que viven del medio rural conocen de primera mano algunas problemáticas que les dificultan desarrollar su proyecto en estos espacios. La caída de los precios de venta de los productos agrarios, el abuso de las grandes empresas multinacionales y los bajos salarios, entre otros, son obstáculos que impiden el desarrollo sostenible del medio rural. Estos y otros problemas pueden ser abordados en la geografía escolar desde el enfoque de los problemas sociales relevantes, que se ha defendido en el apartado anterior, y complementarse con las funciones ecológica, económica y residencial que tiene el medio rural para la sociedad global (Hudault, 2011). Sin embargo, la representación social del medio rural impide comprender y actuar en relación a las inquietudes y deseos que tiene la población que siente estos espacios como propios.

Cuando nos referimos a la representación social del medio rural estamos apelando a una serie de narrativas que pueden acabar obstaculizando la labor del profesorado, si bien es cierto que los docentes también son partícipes de esa representación que afecta a su actitud a la hora de seleccionar los contenidos que imparten en las aulas (Muñoz, 2012). El profesorado no cuenta con una formación específica en la enseñanza del medio rural, un hecho que se agrava con la escasa presencia de la didáctica de las ciencias sociales en los grados de educación, además de que los grados en geografía no cuentan con asignaturas de formación didáctica. Esto se manifiesta en la escasez de investigaciones en la enseñanza de los espacios rurales por parte de los geógrafos españoles (Ruiz, Tula y Molinero, 2017). Desde otros campos de las ciencias sociales se ha demostrado que campo y ciudad se han investigado por separado (Garayo, 1996), un aspecto que también se ha comprobado en el análisis de los contenidos de los libros de texto (Morón y Morón, 2019). El medio rural se identifica con contenidos de corte conservador, algo bastante propio de los libros de texto iberoamericanos (Tonini, Claudino y Souto, 2015), y con un enfoque economicista que lo encuadra en el estudio de las actividades del sector primario bajo el prisma

\footnotetext{
${ }^{4}$ Un ejemplo empírico es la propuesta de cuadernos de alumnado para trabajar desde Educación Primaria, Secundaria y Bachillerato el estudio de la ciudad en relación con los problemas representados en el Museo de Etnología de Valencia, trabajo que se está desarrollando en este curso escolar 2020-21.
} 
del paisaje (Armas, Rodríguez y Macía, 2018; García-Monteagudo, 2019). Estas constataciones explican cómo la idealización del medio rural penetra en el sistema educativo, especialmente en los manuales escolares, y termina obstaculizando la comprensión de los problemas de dicho medio, como se recoge en la Tabla 2.

En la defensa que venimos haciendo sobre la enseñanza de los problemas socialmente relevantes nos interesa comprender las relaciones existentes entre el medio rural y la ciudadanía. La comprensión del medio rural desde la teoría de las representaciones sociales lo convierte en un espacio complejo, en el que las ideas construidas sobre estos espacios vienen determinadas por el contexto social en el que es posible analizar el comportamiento de las personas (Sammut et al., 2015). Este diagnóstico escolar del medio rural no es frecuente en la didáctica de las ciencias sociales (Araya, Souto y Herrera, 2015; García-Monteagudo, 2018), pero es de utilidad para comprender la cosmovisión del medio rural y la promoción de una enseñanza comprometida con las problemáticas reales (Tonge, Mycock \& Jeffery, 2012), en consonancia con las finalidades de esta área de conocimiento (Davies et al., 2018; García Pérez y De Alba, 2007).

\section{Tabla 2}

Elementos que explican la idealización escolar del medio rural

\begin{tabular}{|c|c|c|c|}
\hline & $\begin{array}{l}\text { Narrativas asociadas } \\
\text { a la ruralidad }\end{array}$ & $\begin{array}{l}\text { Aspectos relacionados en los } \\
\text { libros de texto }\end{array}$ & $\begin{array}{l}\text { Percepciones del } \\
\text { alumnado de Educación } \\
\text { Secundaria }\end{array}$ \\
\hline Literatura & $\begin{array}{l}\text { Tópicos: Beatus Ille, } \\
\text { locus amoenus }\end{array}$ & $\begin{array}{l}\text { "(...) el espacio rural es una } \\
\text { reserva totalmente } \\
\text { necesaria para contrarrestar } \\
\text { las secuelas perversas de la } \\
\text { concentración urbana, como } \\
\text { el estrés y la contaminación” } \\
\text { (Fidalgo, Blanco, Picón y } \\
\text { Herrero, 2002, p. 126). }\end{array}$ & $\begin{array}{l}\text { "Porque lo rural es más } \\
\text { tranquilo y calmado } \\
\text { que la ciudad donde } \\
\text { vivo". }\end{array}$ \\
\hline Arte & $\begin{array}{l}\text { Valor estético de los } \\
\text { elementos naturales } \\
\text { del paisaje }\end{array}$ & $\begin{array}{l}\text { ¿Qué tipo de actividades } \\
\text { económicas se desarrollarán } \\
\text { en paraje natural? ¿Y en la } \\
\text { gran ciudad?” (Fidalgo, } \\
\text { Blanco, Picón y Herrero, } \\
\text { 2002, p. 175). }\end{array}$ & $\begin{array}{l}\text { "Creo que en los } \\
\text { espacios rurales hay } \\
\text { elementos naturales: } \\
\text { ríos, montañas, } \\
\text { prados...". }\end{array}$ \\
\hline Cine & Aislamiento social & $\begin{array}{l}\text { "Población rural dispersa es } \\
\text { la constituida por pueblos } \\
\text { pequeños cercanos unos a } \\
\text { otros y con muchas casas de } \\
\text { campo esparcidas entre las } \\
\text { tierras de cultivo (...)" } \\
\text { (Ortega y Roig, 1968, p. 75). }\end{array}$ & $\begin{array}{l}\text { "Asocio espacio rural a } \\
\text { zonas de montaña a las } \\
\text { que se accede por } \\
\text { carreteras empinadas". }\end{array}$ \\
\hline $\begin{array}{l}\text { Publicidad y } \\
\text { redes sociales }\end{array}$ & $\begin{array}{l}\text { Espacios abiertos y } \\
\text { deshabitados }\end{array}$ & $\begin{array}{l}\text { “Puedes distinguir si } \\
\text { predomina el minifundio o el } \\
\text { latifundio?” (Méndez, } \\
\text { Gutiérrez y Guerra, 2010, p. } \\
\text { 149). }\end{array}$ & $\begin{array}{l}\text { "Los espacios rurales } \\
\text { suelen ser zonas con } \\
\text { población anciana y } \\
\text { pocos servicios". }\end{array}$ \\
\hline
\end{tabular}

Fuente: elaboración propia.

La importancia del contexto rural comporta la selección de una información que contempla las motivaciones, creencias, costumbres, comportamientos y expectativas de los sujetos de las áreas rurales, que forma parte de una representación social que se traslada a la escuela rural. 
Entendemos que existe cierto paralelismo entre las dificultades existentes para desarrollar estrategias que integren la escuela rural con las comunidades locales (Rodríguez, 2010; Santiago, 2011) y las innovaciones educativas, ya que alumnado y profesorado no diferencian entre el espacio vivido y el medio local (Romero y Luis, 2008), tal como también ocurre para el caso del medio rural. A pesar de los obstáculos que se vienen señalando para desarrollar una enseñanza problematizadora del medio rural, se ofrecen algunos ejemplos para su consideración por parte del profesorado (Tabla 3). Así podemos observar cómo en diferentes territorios iberoamericanos aparecen problemas sociales que se transforman en contenidos escolares en forma de preguntas (¿Qué dificultades tiene la población inmigrante contratada por grandes propietarios rurales?, ¿Cómo evitar las marginaciones rurales?) que facilitan un proceso de aprendizaje en las aulas con ayuda de los conceptos disciplinares.

\section{Tabla 3}

Relación entre problemas sociales y escolares que afectan al medio rural

\begin{tabular}{|c|c|c|}
\hline Ámbito espacial & Problema social & Problema escolar \\
\hline $\begin{array}{l}\text { Argentina. Programa de } \\
\text { Extensión de Cátedra de } \\
\text { Didáctica de la Geografía }\end{array}$ & $\begin{array}{l}\text { Desigualdades sociales y } \\
\text { económicas entre los } \\
\text { propietarios de tierras y los } \\
\text { trabajadores (inmigrantes). }\end{array}$ & $\begin{array}{l}\text { Acceso a las comunidades } \\
\text { rurales para tomar datos. } \\
\text { ¿Cómo es la vida de los } \\
\text { inmigrantes en los } \\
\text { latifundios? }\end{array}$ \\
\hline $\begin{array}{l}\text { México. Centros de } \\
\text { Integración Popular. }\end{array}$ & $\begin{array}{l}\text { Marginación social de los } \\
\text { habitantes rurales y } \\
\text { dispersión geográfica de las } \\
\text { escuelas. }\end{array}$ & $\begin{array}{l}\text { Exclusión social del alumnado } \\
\text { y las comunidades rurales. } \\
\text { ¿Cómo evitar las } \\
\text { marginaciones rurales? }\end{array}$ \\
\hline $\begin{array}{l}\text { Áreas rurales de Galicia y } \\
\text { norte de Portugal. Proyecto } A \\
\text { ponte. }\end{array}$ & $\begin{array}{l}\text { Mejora de comunicaciones e } \\
\text { infraestructuras entre } \\
\text { espacios urbanos y rurales. }\end{array}$ & $\begin{array}{l}\text { El alumnado presenta } \\
\text { dificultades para hacer un uso } \\
\text { educativo de las TIC. ¿Qué } \\
\text { soluciones para la brecha } \\
\text { digital? }\end{array}$ \\
\hline $\begin{array}{l}\text { Comunidad Valenciana. } \\
\text { Unidades didácticas de la } \\
\text { Cátedra de Cultura Territorial } \\
\text { Valenciana. }\end{array}$ & $\begin{array}{l}\text { Falta de concienciación social } \\
\text { entre las personas y los } \\
\text { territorios. }\end{array}$ & $\begin{array}{l}\text { Diferenciación entre } \\
\text { territorios rurales y urbanos. } \\
\text { ¿Cómo afectan a la vida } \\
\text { cotidiana los servicios de un } \\
\text { lugar? }\end{array}$ \\
\hline
\end{tabular}

Fuente: elaboración propia

De la tabla anterior se pueden extraer algunas interpretaciones para avanzar hacia una didáctica innovadora del medio rural. Las problemáticas de los trabajadores del campo argentino se han investigado por el futuro profesorado que ha realizado un trabajo de campo para comprender los problemas de los productores y las historias de vida de las familias que se dedican a las actividades agrarias (D’Angelo y Lossio, 2011). En México las educadoras apoyan al profesorado de escuelas rurales buscando mejorar las infraestructuras de comunicación y la dotación de recursos para que disminuya la exclusión del alumnado de familias más pobres de las comunidades rurales. El penúltimo ejemplo es el proyecto europeo "A ponte", que permite la colaboración entre estudiantes y profesorado de áreas rurales del norte de Portugal y Galicia (España), con la finalidad de dotar de recursos materiales y organizativos que mejoren la educación y la colaboración entre los miembros participantes (Badía et al., 2004). Sobre estos recursos didácticos, la Cátedra de Cultura Territorial Valenciana ha elaborado unos materiales, 
tanto en Educación Primaria como en Educación Secundaria, para favorecer la comprensión integral del medio y empoderar a la ciudadanía en la toma de decisiones sobre el futuro de los espacios rurales (Peiró, Álvaro, Vílchez y Farinós, 2019).

\subsection{Posibilidades de organización del currículo en torno a problemas sociales}

La introducción de problemas sociales en el aula se plantea como la plasmación de problemas complejos en los que intervienen variables distintas y que no pueden ser entendidas meramente desde la lógica disciplinar. Este enfoque basa su existencia en el modelo crítico, que tiene como objetivo que nuestro alumnado sea capaz de comprender, impugnar y transformar el mundo social. Es por ello que, en la actualidad, tiene poco sentido una enseñanza de contenidos en forma de hechos, conceptos e informaciones de diferentes lugares del mundo sin relación con los grandes problemas de la humanidad (Souto, 2000).

Una organización escolar que conduzca al establecimiento de problemas sociales en el aula demanda reconocer la realidad social como fuente de conocimiento en la escuela, y para su comprensión no se debe recurrir a la atomización disciplinar propuesta por los currículos que presentan la realidad compartimentada por áreas de conocimiento.

La dialéctica entre el problema social y el problema escolar se establece cuando el docente está capacitado para leer críticamente el currículo y tiene la libertad para seleccionar los contenidos y presentárselos al alumnado desde un punto de vista problematizante. De este modo, se presenta una enseñanza de la Geografía y la Historia que promueve la participación ciudadana en la esfera pública, invitando al alumnado a cuestionar y a buscar soluciones sobre los problemas identificados.

Una reciente investigación (Fuertes, Asensi, Fuster y Claudino, 2021) ha permitido demostrar los límites de la formación inicial de los futuros docentes. Aunque se ha observado que el alumnado hace explícita su formación teórica en el paradigma curricular crítico, todavía es insuficiente la difusión de dichas prácticas escolares que conduzcan a un replanteamiento de la organización escolar basada en problemas sociales.

Por su parte Ocampo y Valencia (2019) establecen cinco proposiciones teóricas para la comprensión de la realidad social en la escuela y que pueden ayudar a entender la introducción de los problemas sociales en los currículos escolares. Así, los problemas sociales relevantes posibilitan la integración en la enseñanza de las ciencias sociales, requieren para su estudio la movilización de habilidades cognitivo-lingüísticas, suponen una mirada interdisciplinar, se relacionan con la compresión de la realidad social como finalidad de la enseñanza de las ciencias sociales y suponen un enfoque propio de la didáctica de las ciencias sociales.

A este respecto, encontramos propuestas y materiales curriculares encaminados a trasladar problemas sociales al ámbito escolar iberoamericano. En el caso chileno, uno de los materiales editados por el Ministerio de Educación a través de la editorial SM presenta para $8^{\circ}$ curso Básico el siguiente esquema de trabajo (Landa del Río y Pintor Arratia, 2017):

Unidad 1. Edad Moderna: ¿Qué transformaciones dan origen al mundo moderno? 
Unidad 2. Los españoles llegan a América: ¿encuentro, enfrentamiento o descubrimiento?

Unidad 3. ¿Somos herederos del mundo colonial?

Unidad 4. Ilustración, revolución e Independencia: ¿cambio de edad histórica?

Unidad 5. ¿Qué criterios se utilizan para definir una región?

En el ámbito español encontramos las propuestas de diferentes grupos de renovación pedagógica. Así en el caso de Gea-Clío contamos con los materiales editados en los años 90 del siglo pasado encaminados a introducir en el aula problemas sociales. Como ejemplo, en Secundaria Obligatoria: El tiempo libre, ¿Por qué se mueren los ríos?, Mi mundo y el Globo donde vivo yo, La escuela ayer y hoy, entre otros.

Asimismo, el Grupo de Investigación e Innovación en el Currículum de Ciencias Sociales (GRICSO) de la Universidad Autónoma de Barcelona propone, en Secundaria, un proyecto que se articula a partir de un hilo conductor: el proceso que lleva de la hominización a la humanización. Presentando una secuenciación basada en diez temas:

1. Somos polvo de estrella: ¿qué nos vincula al Universo?

2. Un planeta con mucha suerte: ¿por qué la superficie de nuestro planeta es un escenario cambiante?

3. El misterio de la vida: ¿qué compartimos con los demás seres vivos?

4. El planeta humano: ¿qué nos hace humanos?

5. La agricultura: ¿la Tierra nos alimenta a todos?

6. Comunicaciones y mercados: ¿un camino hacia el progreso?

7. La industria y la tecnología: ¿la industrialización, una mejora para todos?

8. Las sociedades humanas: ¿existe la igualdad de derechos?

9. La democracia: ¿el mejor de los sistemas políticos posible?

10. Un mundo sostenible y justo: ¿qué futuro queremos?

La plasmación de los ejemplos anteriores conlleva la introducción de problemas sociales en el aula, lo que nos permite acercar al alumnado situaciones conflictivas que pueden animarle a participar en el seguimiento de las actividades docentes. Su introducción supone organizar una secuencia de actividades para facilitar el aprendizaje. De todo el espectro de secuencias, creemos oportuno citar la aplicación del Aprendizaje Basado en Problemas (ABP) (Cachinho, 2011; García de la Vega, 2010), la cual nos ofrece una estructura de trabajo que nos permite introducir en el aula el tratamiento de problemas sociales.

En síntesis, esta metodología se basa en la utilización de problemas socialmente relevantes formulados adecuadamente para motivar a los estudiantes a identificar, investigar y aprender los conceptos y principios que necesitan para resolverlos. De este modo, se promueve el aprendizaje significativo y la investigación escolar, además de suscitar el interés por aprender del alumnado y promover la adquisición de competencias. 
Asimismo, el ABP fomenta el desarrollo y adquisición de habilidades cognitivas, y que, teniendo en cuenta la taxonomía de Bloom, revisada por Anderson y Krathwohl (2001), promueven procesos cognitivos elevados como los descritos por Barrows (1986): 1) estructurar el conocimiento, 2) desarrollar procesos de razonamiento, 3) desarrollar destrezas de aprendizaje, 4) fomentar la motivación para el aprendizaje, 5) desarrollar el trabajo en grupo.

En publicaciones anteriores (Fuster, 2015) presentamos, a modo de ejemplo, una experiencia llevada a cabo en un grupo de 3ํㅡㄹ de ESO que surgió de las inquietudes del alumnado. Por aquel entonces, la presión informativa de los medios de comunicación en relación a la entrada de migrantes ilegales como resultado del salto de la valla era recurrente en los medios de comunicación. Como consecuencia se programó una experiencia que consistía en la resolución de un problema en el que abordar las cuestiones más relevantes relacionadas con el tema de los procesos migratorios.

A partir del trabajo desarrollado con el alumnado, se demostró que, gracias a la introducción de problemas sociales en el aula, el alumnado no solo adquirió los contenidos propiamente dichos, sino que se fomentó el desarrollo de la autonomía personal, fomentando que los estudiantes crearan una opinión propia con argumentos para defenderla, además de crear el contexto para demostrar y defender sus ideas.

En esta ocasión, la problematización del currículo permitió introducir en el aula y reproducir a pequeña escala los problemas reales con los que el estudiante se enfrenta en su vida cotidiana.

En otro ejemplo no publicado, se presentó el siguiente problema: tras el estudio del nomenclátor del callejero de una localidad de la provincia de Valencia se detectó la falta de calles con nombres de personajes femeninos. A partir de la presentación del problema se ofreció a los estudiantes la posibilidad de poner en conocimiento de la entidad local los resultados del estudio realizado. En esta ocasión, como señalan Ospina y Valencia (2019), se trataba de introducir en el aula un fenómeno que se encontraba sometido a un juicio social, pero que adquiría un sentido especial en el momento de introducirlo en el aula, transformando un problema social en escolar. De este modo, los estudiantes, al observar el fenómeno social están obligados a elaborar explicaciones presentes en la realidad.

En este sentido, el enfoque de dicho problema social se convirtió en el camino para que desde el aula se apostara por la comprensión de la realidad social. No solo contribuyendo a la formación de un pensamiento crítico, sino también al ofrecimiento de participación ciudadana, estableciendo relaciones entre entidades públicas (ayuntamiento y centro educativo).

\section{Conclusiones}

La problematización de los acontecimientos sociales nos ha facilitado la elaboración de unidades didácticas que relacionan el estudio escolar con la comprensión de los fenómenos cotidianos, lo que repercute en la motivación del alumnado respecto al aprendizaje de los hechos y conceptos que se sintetizan como contenidos curriculares. Además, dicha interpretación de los hechos permite relacionar la investigación observante en las aulas con los estudios académicos, paliando así las dificultades de relación entre las instituciones educativas del sistema básico 
escolar y del universitario (Estepa, 2019; Prats, 2020). Es evidente que, sin un compromiso social y una reflexión ontológica sobre el saber escolar, es difícil precisar unas metas para avanzar metodológicamente. Se trata de una tarea colectiva para romper el aislamiento de cada clase y centro escolar, que busca alianzas entre investigación e innovación, como se hizo desde los proyectos curriculares que se han analizado.

Estas iniciativas las hemos encontrado en los grupos de innovación de Educación Secundaria en España, en los años finales del siglo XX, que llevaban implícita la definición del conocimiento profesional del/la docente, como se refleja en los estudios realizados en el seno del grupo IRES, en su propuesta de "conocimiento escolar 'deseable', a modo de hipótesis de intervención didáctica" (García Pérez, 2015, p. 50). La organización institucional del saber escolar configuraba un campo de conocimiento con reglas y normas que condicionaban el trabajo personal en las aulas de enseñanza básica.

La manera alternativa de interpretar el currículo afectó al cuestionamiento del saber académico, impugnándose la singularidad natural de las disciplinas. Un camino que implica una profunda revisión epistemológica de las materias escolares y de su incidencia en la formación del profesorado (Jiménez y Felices de la Fuente, 2018) y en el análisis de las aportaciones disciplinares, como se ejemplificó, en su momento, en el caso de la geografía (García Pérez, 2011).

Las evidencias de la experimentación de las unidades didácticas de los grupos de innovación nos indicaron que la expansión de este modelo de enseñanza sobre problemas relevantes es lenta. Por eso se impone el uso de estrategias metodológicas que nos permitan avanzar con cautelas (De Alba y Porlán, 2020).

En todo caso, los estudios que mostramos en este artículo nos indican la posibilidad de impugnar contenidos clásicos que suponen la idealización de un territorio, en este caso el medio rural. Pero también la posibilidad de ejercer la participación ciudadana con ejemplos en los fenómenos migratorios o en la toponimia cultural del callejero urbano. Una tarea que también se difunde, entre otros casos, con el proyecto Nós Propomos, como señalamos en este mismo artículo.

El trabajo con cuestiones relevantes supone, pues, no solo un desafío para los docentes de los Grados de Educación Infantil y Primaria, así como del Máster de Secundaria, sino también para el propio alumnado universitario, que ha generado unas rutinas y prejuicios en consonancia con las representaciones sociales del sistema escolar y de la figura concreta del/la docente. Por eso se generan incertidumbres y conflictos en las aulas universitarias cuando se aborda esta metodología, pues se está impugnando una manera hegemónica de enseñar, que no se suele cuestionar. Romper con estas inercias constituye un camino largo y una constancia en la autoestima del modelo de profesor que innova e investiga.

La dialéctica que hemos establecido entre problemas sociales y escolares nos permitirá no sólo cuestionar los contenidos culturales enciclopédicos, que se presentan como "naturales" y propios de cada materia ${ }^{5}$, sino también cuestionar el papel de la enseñanza escolar ante la participación ciudadana. $Y$, en este sentido, es donde cobra mayor validez la propuesta de una metodología que se pueda desarrollar en las aulas y que facilite elementos y posibilidades para la investigación educativa. Con ello se podrán obtener pruebas de la mejora sustancial del

5 Tanto en la línea de las representaciones sociales como en los estudios sociogenéticos de las materias escolares. 
aprendizaje para entender críticamente la sociedad y poder participar de forma consciente en la ciudadanía.

\section{Referencias bibliográficas}

Anderson, L. W. y Krathwohl, D. R. (2001). A Taxonomy for Learning, Teaching, and Assessing: A Revision of Bloom's Taxonomy of Educational Objectives. London: Longman.

Araya, F., Souto, X.M. y Herrera, Y. (2015). El espacio geográfico, una construcción escolar. Un estudio de caso: los alumnos del valle del Limarí (Chile). Scripta Nova, Revista Electrónica de $\begin{array}{llllll}\text { Geografía } & y & \text { Ciencias } & \text { Sociales, } & \text { vol. } & \text { XIX, }\end{array}$ http://revistes.ub.edu/index.php/ScriptaNova/article/view/15108/18311

Armas, F.X., Rodríguez, F. y Macía, X.C. (2018). La olvidada geografía rural en el currículo y manuales de la educación secundaria. REIDICS, Revista de Investigación en Didáctica de las Ciencias Sociales, 3, 4-19. https://doi.org/10.17398/2531-0968.03.04

Badía, A., Bautista, G., Guasch, T., Sangrà, A. y Sigalés, C. (2004). La integración escolar de las TIC: el Proyecto Ponte dos Brozos. Universitat Oberta de Cataluña. Recuperado de https://www.uoc.edu/dt/esp/badia0904.pdf

Barrows, H. S. (1986). A Taxonomy of problem-based learning methods. Medical Education, 20, 481-486. https://doi.org/10.1111/j.1365-2923.1986.tb01386.x

Cachinho, H. (2011). Inovaçôes didácticas e ensino da Geografía: do potencial da aprendizagem baseada em problemas. En J. J. Delgado, M. L. de Lázaro y M. J. Marrón Gaite (Coords.), Aportaciones de la Geografía en el aprendizaje a lo largo de la vida (pp. 242-257). Congreso Ibérico de Didáctica de la Geografía. Universidad Complutense de Madrid.

De Alba-Fernández, N. y Porlán, R. (2020). La metodología de enseñanza. En N. De Alba-Fernández y R. Porlán (Coords.), Docentes universitarios. Una formación centrada en la práctica (pp. 3754). Madrid: Morata.

D'Angelo, M.L. y Lossio, O. (2011). Innovar en la enseñanza de geografía rural en la escuela secundaria: propuesta de desarrollo profesional docente. Revista Geográfica de América $\begin{array}{llll}\text { Central, II } & \text { Semestre, }\end{array}$ https://www.revistas.una.ac.cr/index.php/geografica/article/view/2733

Davies, I., Ching, L.I., Kiwan, D., Peck, C., Peterson, A., Sant, E. \& Waghid, Y. (2018). The Palgrave Handbook of Global Citizenship and Education. Palgrave Macmillan. https://doi.org/10.1057/978-1-137-59733-5

Domingos, M. (2000). "Habitus e representações sociais: questões para o estudo de identidades coletivas. En A.S.P. Moreira y D.C. Oliveira (Orgs.), Estudos interdisciplinares de representação social (pp. 117-159). Goiânia: AB, 2ª edición.

Domingos M. y Diniz L. (2019). Representações Sociais, Sens Pratique, Poder Simbólico e o Processo de Construção do Ser Docente. Arxius de Ciències Socials, 41, 43-64.

Duarte, 0. (2015) La enseñanza de la historia en la educación secundaria: innovación, cambio y continuidad. Tesis doctoral. Universidad de Sevilla. https://search.ebscohost.com/login.aspx?direct=true\&db=edsbas\&AN=edsbas.79DD46B3 \&lang=es\&site=eds-live\&scope $=$ site 
Estepa-Giménez, J. (2019). Investigar para innovar: el caso del Ámbito de Investigación de las sociedades actuales e históricas, REIDICS, Revista de Investigación en Didáctica de las Ciencias Sociales, 4, 4-19.

Fidalgo, C., Blanco, J., Picón, J. y Herrero, J. (2002). Ciencias Sociales, Geografía. Proyecto Zenit. Comunidad Valenciana. $3^{\circ}$ curso de Educación Secundaria. SM.

Fuertes, C., Asensi, A., Fuster, C. y Claudino, S. (2021). Socially acute questions and critical citizenship in trainee geography and history teachers: From theory to classroom observation. En C. Gómez, P. Miralles y R. López (coord.). Handbook of Research on Teacher Education in History and Geography (cap. 11). Berlin: Peter Lang.

Fuster García, C. (2015). Visiones de un mundo en crisis. La problematización del currículum de geografía como estrategia didáctica. En A.M. Hernández, C.R. García y J.L. de la Montaña (coords.), Una enseñanza de las ciencias sociales para el futuro: Recursos para trabajar la invisibilidad de personas, lugares y temáticas (pp.155-162). Cáceres: AUPDCS y Universidad de Extremadura.

Garayo, J.M. (1996). La sociedad rural en el final de siglo. Inguruak. Revista Vasca de Sociología y Ciencia Política, 16, 61-80.

García de la Vega, A. (2010). Aplicación Didáctica del aprendizaje basado en problemas al análisis geográfico. Revista de Didácticas Específicas, 2, 43-60.

García-Monteagudo, D. (2018). Análisis metodológico de las representaciones sociales del medio rural entre estudiantes de Educación Secundaria: un estudio de caso. En V. Peris, D. Parra y X.M. Souto (coord.), Repensamos la geografía y la historia para la educación democrática (pp. 75-87). Valencia: Nau Llibres.

García-Monteagudo, D. (2019). Tradiciones en la enseñanza del medio rural desde una perspectiva iberoamericana: análisis de los libros de texto de España y Brasil. En M.J. Hortas, A. Dias y N. de Alba (Eds.), Enseñar y aprender didáctica de las ciencias sociales: la formación del profesorado desde una perspectiva sociocrítica (pp.50-58). Asociación Universitaria del Profesorado de Didáctica de las Ciencias Sociales.

García-Monteagudo, D., Fuster, C., y Souto, X.M. (2017). Estrategias de resolución de problemas en la formación docente. Estudios de casos. REIDICS, Revista de Investigación en Didáctica de las Ciencias Sociales, 1, 132-147.

García Pérez, F.F. (2011). Geografía, Problemas Sociales y conocimiento escolar. Anekúmene, 2, 621.

García Pérez, F.F. (2015). El conocimiento escolar en el centro del debate didáctico. Reflexiones desde la perspectiva docente. Con-Ciencia Social, 19, 49-62. Recuperado de http://www.fedicaria.org/concSocial/conc_19.htm

García Pérez, F.F. y De Alba, N. (2007). Educar en la participación como eje de una educación ciudadana. Reflexiones y experiencias. Didáctica Geográfica, 9, 243-258. https://didacticageografica.agegeografia.es/index.php/didacticageografica/article/view/ $16 / 16$

González-Monfort, N. y Santisteban Fernández, A. (2020). Alfabetización crítica para interpretar problemas sociales. Iber: Didáctica de las ciencias sociales, geografía e historia, 99, 39-45. 
Grupo Cronos (1997). El conocimiento socialmente relevante: la enseñanza de las ciencias sociales entre problemas y disciplinas. Aula de Innovación Educativa, 61, 6-8.

Hudault, J. (2011). La protección jurídica del territorio rural. En E. Muñiz (coord.), Un marco jurídico para un medio rural sostenible (pp. 71-80). Madrid: Ministerio de Medio Ambiente y Medio Rural y Marino.

Jiménez, Má.D. y Felices de la Fuente, M. (2018). Cuestiones socialmente vivas en la formación inicial del profesorado: la infancia refugiada siria como problemática. REIDICS, Revista de Investigación en Didáctica de las Ciencias Sociales, 3, 87-102.

Landa del Río, L. y Pinto Arratia, V. (2017). Historia, Geografía y Ciencias Sociales. 8o Básico. Departamento de Estudios Pedagógicos de Ediciones SM.

Méndez, R., Gutiérrez, J. y Guerra, A. (2010). Ciencias Sociales, Geografía. 3ํㅡㄹ. ESO.

Merchán, F.J. y García Pérez, F.F. (1997). El tratamiento de problemas de nuestro mundo en la enseñanza obligatoria. Aula de Innovación Educativa, 61, 9-12.

Morón, Ma .C. y Morón, H. (2019). El tratamiento del Medio Rural en el Currículum Oficial de Secundaria en la Última Década. En X.C. Macía, F.X. Armas y F. Rodríguez (Eds.), La reconfiguración del medio rural en la sociedad de la información. Nuevos desafíos en la educación geográfica (pp. 896-906). Santiago: Andavira Editora.

Muñoz, I. (2012). La ruralidad: análisis de las representaciones del profesorado y propuesta para una enseñanza aprendizaje del compromiso con la ruralidad, aplicada en la región de Maule (Chile). Tesis doctoral inédita. Universitat Autònoma de Barcelona.

Ocampo Ospina, L. y Valencia Carvajal, S. (2019). Los problemas sociales relevantes: enfoque interdisciplinar para la enseñanza integrada de las ciencias sociales, REIDICS, Revista de Investigación en Didáctica de las Ciencias Sociales, 4, 60-75.

Ortega, D. y Pagès, J. (2017). Las representaciones sociales de los problemas contemporáneos en estudiantes de magisterio de Educación Primaria. Investigación en la Escuela, 93, 1-15. https://doi.org/10.12795/IE.2017.i93.01

Ortega, R. y Roig, J. (1968). Geografía de España. 1o curso de Bachillerato. Ed. Vicens Vives.

Pagès, J. y Santisteban, A. (2011). Les Qüestions socialment vives i l'ensenyament de les ciències socials. Servei de Publicacions. Universitat Autònoma de Barcelona.

Pedrouzo, O., Díaz, A. y López Facal, R. (1995). Algunas dudas sobre la elaboración de unidades didácticas. Iber: Didáctica de las ciencias sociales, geografía e historia, 4, 75-86.

Peiró, E., Álvaro, N., Vílchez, A. y Farinós, J. (2019). Hacia una nueva cultura territorial desde la educación básica obligatoria. La experiencia de las unidades didácticas “¿Qué conozco de mi territorio?" de la Cátedra de Cultura Territorial Valenciana. En XXVI Congreso de la Asociación Española de Geografía, Crisis y espacios de oportunidad. Retos para la Geografía (pp.143-158). Asociación Española de Geografía y Departamento de Geografía de la Universitat de València.

Prats Cuevas, J. (2020). Didáctica de la historia en secundaria y en la universidad. Dos mundos que viven de espaldas. Íber. Didáctica de las Ciencias Sociales, Geografía e Historia, 100, 10-14.

Rodríguez, A. (2010). Familia rural, valores ambientales y sostenibilidad. Un estudio de educación ambiental en el condado norte de Huelva. En: M. Junyentut y L. Cano (Coords.), Investigar 
para avanzar en educación ambiental (pp. 181-199). Organismo Autónomo Parques Nacionales. Madrid: Ministerio de Medio Ambiente y Medio Rural y Marino.

Romero, J. y Luis, A. (2008). El conocimiento sociogeográfico en la escuela: las tensiones inherentes a la transmisión institucionalizada de cultura y los dilemas de la educación para la democracia en este mundo globalizado. Scripta Nova, Revista Electrónica de Geografía y Ciencias Sociales, XII (270) (123). http://www.ub.edu/geocrit/sn/sn-270/sn-270-123.htm

Ruiz, A.R., Tula, A.F., y Molinero, F. (2017). La enseñanza de la geografía rural en los estudios universitarios de grado en España: temáticas clave, organización y metodologías de trabajo actuales. Biblio 3W, Revista Bibliográfica de Geografía y Ciencias Sociales, vol. XXII, 1198. http://www.ub.edu/geocrit/b3w-1198.pdf

Sammut, G., Andreouli, E., Gaskell, G., \& Valsiner, J. (2015). Social representations: a revolutionary paradigm? In G. Sammut, E. Andreouli, G. Gaskell \& J. Valsiner (Eds.), Cambridge Handbook of Social Representations (pp. 3-11). Cambridge University Press. https://doi.org/10.1017/CB09781107323650.003

Santiago, J. A. (2011). Educación rural y la enseñanza de la geografía. Sapiens: Revista Universitaria De Investigación, 12(2), 64-76. https://www.redalyc.org/pdf/410/41030368005.pdf

Santidrián, V.M. y López Facal, R. (2011). Los «conflictos sociales candentes» en el aula. Íber: Didáctica de las Ciencias Sociales, Geografía e Historia, 69, 8-20.

Santisteban, A. (2019). La enseñanza de las Ciencias Sociales a partir de problemas sociales o temas controvertidos: estado de la cuestión y resultados de una investigación. El Futuro del Pasado, 10, 57-79.

Souto, X.M. (2000). La didáctica de la geografía: dudas, certezas y compromiso social de los docentes. XVI Congreso Colombiano de Geografía. Memorias (pp. 141-152). Cali: ACOGE.

Souto, X.M. y Ramírez, S. (1996). Enseñar Geografía o educar geográficamente a las personas. Iber: Didáctica de las ciencias sociales, geografía e historia, 9, 15-26.

Souto, X.M., García-Monteagudo, D. y Fuster, C.. (2016). Explicamos las identidades ciudadanas escolares. En R. López Facal (Ed.), Ciencias sociales, educación y futuro. Investigaciones en Didáctica de las Ciencias Sociales. VII Simposio Internacional de Didáctica de las Ciencias Sociales en el ámbito iberoamericano (pp. 992-1005). Universidade de Santiago de Compostela.

Souto, X.M., García-Monteagudo, D. y Fuster, C. (2018). Una propuesta metodológica para analizar la representación social del saber escolar. En V. Peris, D. Parra y X.M. Souto (Eds.), Repensamos la Geografía e Historia para la educación democrática (pp. 49-74). Valencia: Nau Llibres.

Tonge, J., Mycock, A. \& Jeffery, B. (2012). Does citizenship education make young people betterengaged citizens? Political Studies, 60(3), 578-602. https://doi.org/10.1111/j.14679248.2011.00931.x

Tonini, I.M., Claudino, S. y Souto, X.M. (2015). Manuais escolares de Geografia de Brasil, Espanha e Portugal: quais as inovações didáticas para o ensino de Geografia? En R. Sebastiá y E.Ma. Tonda (Coords.), Investigar para innovar en la enseñanza de la Geografía (pp.191-205). Alicante: CEE Limencop. 